\title{
JAZF1-SUZ12 endometrial stromal sarcoma forming subserosal masses with extraordinary uptake of fluorodeoxyglucose on positron emission tomography: a case report
}

Koto Fujiishi', Shigenori Nagata ${ }^{1 *} \mathbb{B}$, Rieko Kano ${ }^{1}$, Chiaki Kubo ${ }^{1}$, Maasa Shirayanagi ${ }^{2}$, Megumi Ozaki², Takashi Yamamoto ${ }^{2}$, Katsuyuki Nakanishi ${ }^{3}$, Shoji Kamiura ${ }^{4}$ and Shin-ichi Nakatsuka ${ }^{1}$

\begin{abstract}
Background: Low-grade endometrial stromal sarcoma (ESS) is rare mesenchymal neoplasm, recently specified as harboring JAZF1-SUZ12 rearrangement. Typical JAZF1-SUZ12 ESS is slow growing, in which high uptake of fluorodeoxyglucose (FDG) on positron emission tomography (PET) and subserosal masses are quite unusual.

Case presentation: A 69-year-old Japanese woman complained of urinary incontinence. Pelvic magnetic resonance imaging showed uterine lesions composed of (1) a $9 \times 8 \times 7-\mathrm{cm}$ mass protruding from the right-anterior wall, (2) a 4.5$\mathrm{cm}$ mass attached to the right-posterior wall, and (3) a $6.5-\mathrm{cm}$ intramural mass in the fundus. FDG-PET demonstrated maximum standardized uptake value of 13.28 confined to the two subserosal masses ( 1 \& 2 ) in contrast to no uptake of the intramural mass (3). She was diagnosed with a high-grade uterine sarcoma concomitant with leiomyomas and underwent total hysterectomy with bilateral salpingo-oophorectomy and pelvic lymphadenectomy. The removed uterus had three tumors - two in the right-anterior and right-posterior subserosa, respectively, and the remaining in the fundal myometrium. Microscopically, the three tumors shared morphologic features characterized by neoplastic cells similar to proliferative-phase endometrial stromal cells, in which neither round-cell component, pleomorphism, nor high mitotic activity was recognized. Nuclear cyclin D1 immunostaining was identified 50\% of neoplastic cells in the two subserosal tumors (1 \&2) whereas < 1\% positive cells in the intramural component (3). Reverse transcriptasepolymerase chain reaction showed the same-sized electrophoretic bands indicating JAZF1-SUZ12 gene fusion shared by the three uterine tumors and a focal tumor extension into the extrauterine vein. The patient is alive without evidence of recurrence at 14 months after surgery.
\end{abstract}

Conclusions: Pathologists and clinicians should not exclude the possibility of JAZF1-SUZ12 ESS even when uterine subserosal masses demonstrate extraordinary FDG uptake on PET. Molecular analysis is helpful for diagnostic confirmation of JAZF1-SUZ12 ESS with a complex growth pattern.

Keywords: Endometrial stromal sarcoma, JAZF1-SUZ12, Subserosal mass, Cyclin D1, Positron emission tomography

\footnotetext{
* Correspondence: nagata-si@mc.pref.osaka.jp

'Department of Diagnostic Pathology and Cytology, Osaka International

Cancer Institute, Osaka, Japan

Full list of author information is available at the end of the article
}

(c) The Author(s). 2019 Open Access This article is distributed under the terms of the Creative Commons Attribution 4.0 International License (http://creativecommons.org/licenses/by/4.0/), which permits unrestricted use, distribution, and reproduction in any medium, provided you give appropriate credit to the original author(s) and the source, provide a link to the Creative Commons license, and indicate if changes were made. The Creative Commons Public Domain Dedication waiver (http://creativecommons.org/publicdomain/zero/1.0/) applies to the data made available in this article, unless otherwise stated. 


\section{Background}

Endometrial stromal tumors are uncommon; they account for less than $10 \%$ of uterine mesenchymal neoplasms and less than $2 \%$ of all uterine tumors [1]. They are sorted by the 2014 World Health Organization (WHO) classification into four categories: endometrial stromal nodule, low-grade endometrial stromal sarcoma (ESS), high-grade ESS, and undifferentiated uterine sarcoma [2]. Low-grade ESS is the second most common mesenchymal malignancy of the uterus and recently characterized by harboring the genetic rearrangement $\mathrm{t}$ $(7 ; 17)(\mathrm{p} 15 ; \mathrm{q} 21)$ resulting in JAZF1-SUZ12 gene fusion [3]. Low-grade ESS is typically slow growing, in which high uptake of fluorodeoxyglucose (FDG) on positron emission tomography (PET) and subserosal masses are much less common than in leiomyosarcomas. Here, we describe a woman with JAZF1-SUZ12 ESS of the uterine corpus forming two sizeable subserosal masses other than a conventional intramural component, the two former of which exhibited exceptionally high FDG uptake on PET/computed tomography indicating distinctive heterogeneity in proliferative activity within a single tumor.

\section{Case presentation}

\section{Clinical course}

A 69-year-old Japanese postmenopausal (gravida 3, para 3) woman went to her primary physician complaining of urinary incontinence 3 months before admission to our institute. She was suspected of having a uterine mass, which had been growing up in size of $8 \mathrm{~cm}$ to $9 \mathrm{~cm}$ during prior 2 months. Her medical history was unremarkable. She had no past history of neoplasms and no family history of cancer. Blood testing showed slightly elevated levels of lactate dehydrogenase (LDH) $269 \mathrm{U} / \mathrm{L}$ (normal range: 124-222 U/L) and cancer antigen (CA) $12546 \mathrm{U} / \mathrm{mL}$ (cutoff value: $35 \mathrm{U} / \mathrm{L}$ ). CA 19-9 level was within normal limits. The pelvic examination and transvaginal ultrasonography revealed a fist-sized uterine corpus without abnormalities in other reproductive organs. The cervical cytology was normal. Pelvic magnetic resonance imaging demonstrated a $9 \times 8 \times 7-\mathrm{cm}$ mass protruding from the right-anterior wall of the uterine corpus that showed heterogenous high intensity on T2weighted images, and high intensity on diffusionweighted images (DWI), coexisting with a $4.5 \times 3.5-\mathrm{cm}$ mass attached to the right-posterior wall of the uterus and a $6.5 \times 4.5-\mathrm{cm}$ intramural mass in the fundus, both demonstrating slightly high intensity on DWI (Figs. 1ac). On ${ }^{18}$ F-FDG-PET, maximum standardized uptake value was 13.28 , confined to the tumors located in the uterine right-anterior and right-posterior wall, in sharp contrast to no uptake in the intramural tumor (Fig. 1d). There was no evidence of distant disease. She was radiologically diagnosed with a high-grade uterine sarcoma and concomitant leiomyoma, of which preoperative biopsy was not performed. She underwent total abdominal hysterectomy with bilateral salpingo-oophorectomy and
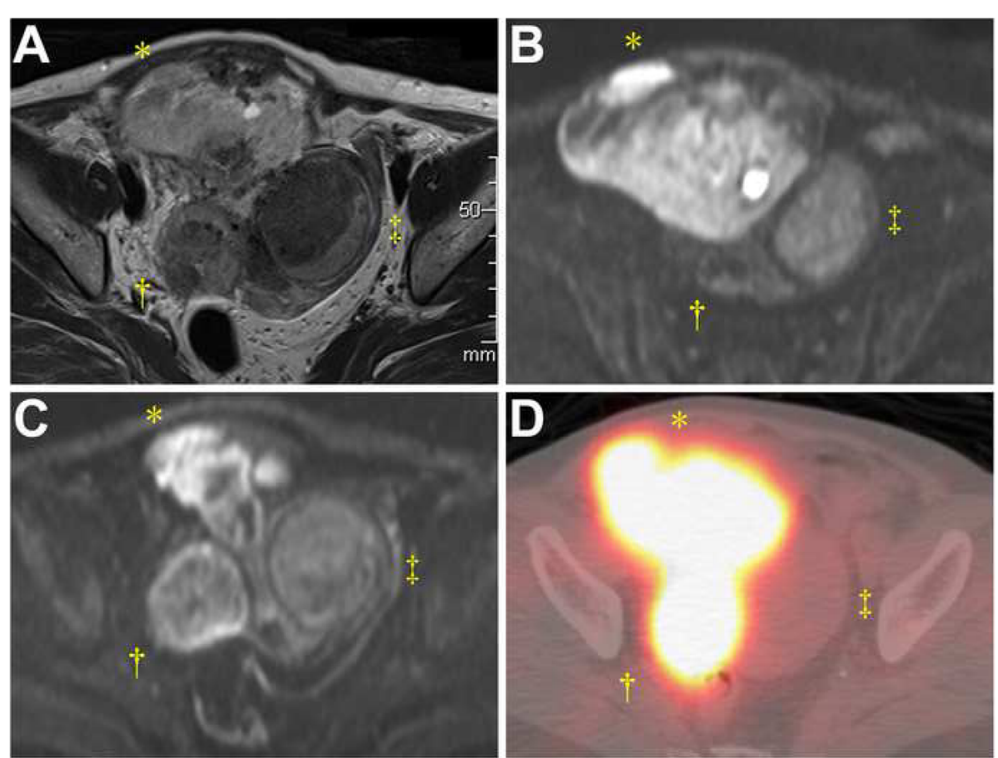

Fig. 1 Imaging of the uterine tumor composed of three components. a Pelvic axial T2-weighted magnetic resonance imaging disclosing a mass with heterogenous, mixed low and high intensity $\left(^{*}\right)$ and another mass with mostly low intensity ( $\dagger$ ) attached to the right-anterior and rightposterior serosa, respectively, and the remaining low-intensity intramural mass in the fundus ( $\neq$ ). $\mathbf{b}$ and $\mathbf{c}$ Diffusion-weighted images showing high intensity in the right-anterior tumor $\left(^{*}\right)$ and slightly high intensity in the right-posterior ( $\dagger$ ) and the fundal ( $\neq$ ) tumors (d) Positron emission tomography/computed tomography fusion image demonstrating high ${ }^{18}$ F-fluorodeoxyglucose uptake of the right-anterior and right-posterior tumors $(*,+)$ in contrast to no uptake of the fundal tumor $(\neq)$ 
pelvic lymphadenectomy, followed by an uneventful postoperative course. She received no adjuvant therapy, being alive without evidence of recurrence at 14 months after the surgery.

\section{Pathologic examination}

Macroscopically, the removed uterus revealed three separate masses, two of which were located on the uterine right-anterior and right-posterior serosa, respectively, with the remaining mass confined to the fundal myometrium (Fig. 2a). Appearances of the cut surfaces varied by lesion, which were yellow to tan or grayish white coloured, solid or lobulated, accompanied by extensive degeneration and focal hemorrhage in the largest tumor (Fig. 2b). On microscopic examination, the three tumors shared the morphologic feature of proliferating cells having round to ovoid nuclei with a high nuclear-to- cytoplasmic ratio, similar to that of proliferative-phase endometrial stromal cells, with somewhat different cytohistologic features including intercellular edema and fibromyxoid changes in the right-anterior and right-posterior tumors, respectively (Figs. 2c-e). Neither round-cell component nor pleomorphism was identified, and mitoses did not exceed 3 counts per 10 high-power-fields (HPF) anywhere in the three tumors. Vascular permeation was prominent and "worm-like" at the periphery of the fundal tumor, accompanied by focal extrauterine extension of the tumor into the pelvis, swelling $2.0 \mathrm{~cm}$ in size (Fig. 2f). Neither lymphatic permeation nor lymph-node metastasis was observed. The uterine cervix and both appendages were not involved by the tumor. The histologic diagnosis was Stage IB low-grade ESS according to the WHO and International Federation of Gynecology and Obstetrics 2014 Classification.
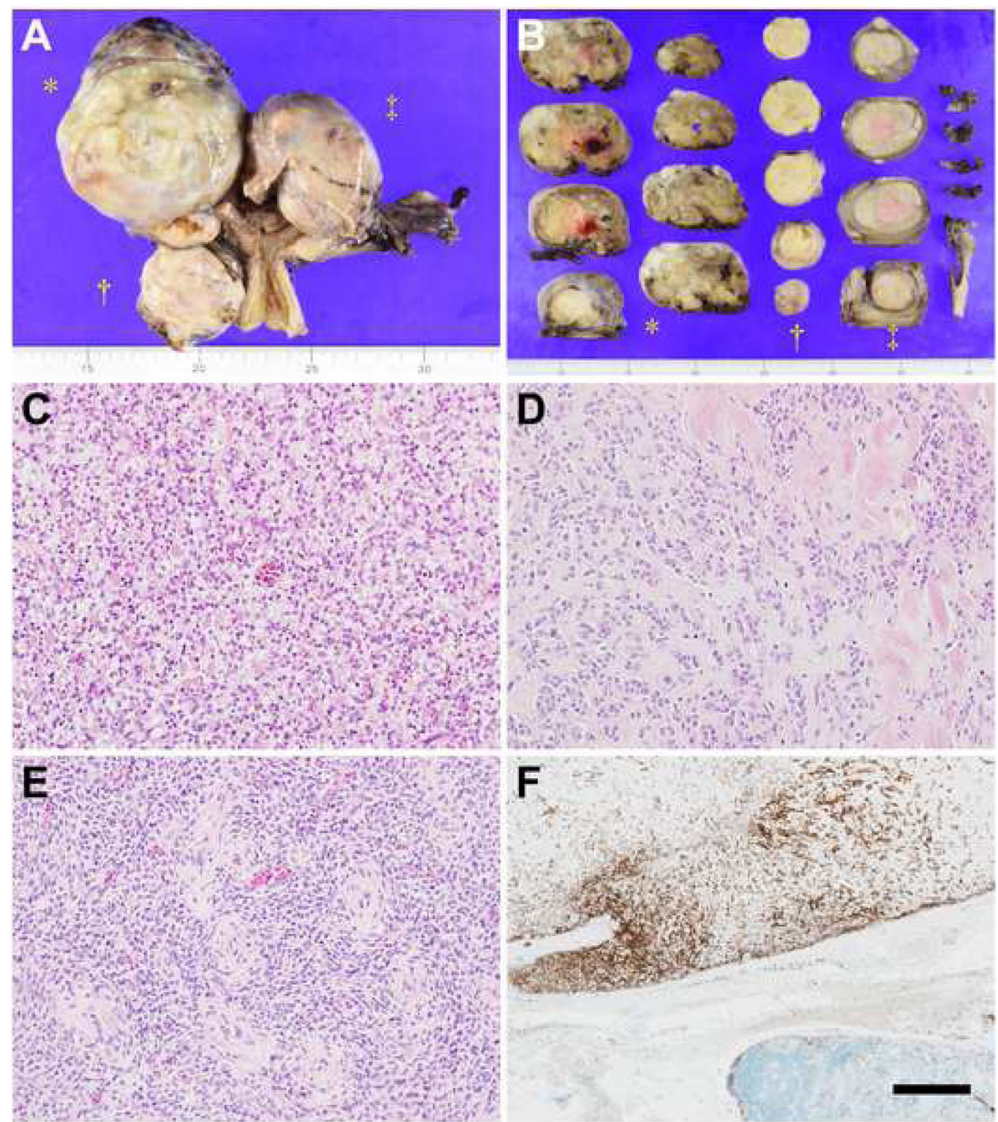

Fig. 2 Photographs of the surgically removed uterine tumor. a Three uterine masses separately located on the right-anterior $\left(^{*}\right)$ and rightposterior $(\dagger)$ serosa and within the fundal myometrium ( $\neq$ ) of $9.5 \mathrm{~cm}, 4.5 \mathrm{~cm}$, and $6.5 \mathrm{~cm}$ in largest diameter, respectively. $\mathbf{b}$ Cut surfaces of the uterine tumor by lesion, which were heterogenously tan to yellow with focal hemorrhage $\left(^{*}\right)$, white with yellow plaques $(\dagger)$, and pale-gray with intratumoral mosaic demarcation (¥). c-e Cytohistologic variation by lesion. (c) Uniform cells having round to ovoid nuclei and scant cytoplasm proliferating with intercellular edema (hematoxylin and eosin [HE] stain, $\times 100$ ). $\mathbf{d}$ Tumor cells arranged in trabecular cords admixed with abundant fibromyxoid matrix $(\mathrm{HE}, \times 100)$. e Classic morphology of low-grade endometrial stromal tumor characterized by numerous whorls around small vessels $(\mathrm{HE}, \times 100)$. f Tumor extension into the extrauterine vein positive for CD10 (the upper area), adjacent to nonmetastatic pelvic lymph nodes (immunohistochemical [IHC] stain; bar, $0.5 \mathrm{~mm}$ ) 


\section{Immunohistochemical study}

All the three tumors were positive for CD10, Wilms' tumor 1, estrogen receptor, progesterone receptor, and androgen receptor in most areas, and focally positive for $\alpha$-smooth muscle actin and h-caldesmon. Cyclin D1 nuclear staining was positive in $50 \%$ of neoplastic cells with weak to moderate intensity in the two subserosal tumors whereas the fundal tumor showed $<5 \%$ Cyclin D1 positive tumor cells (Figs. 3a-c). Ki-67 labeling indices of the right-anterior, right-posterior, and fundal tumors were 10,10 , and $3 \%$, respectively.

\section{Molecular analysis}

Reverse transcriptase-polymerase chain reaction (RTPCR) was performed, using formalin-fixed and paraffinembedded tissues obtained from each uterine tumor and the extrauterine venous extension. Complementary DNA was produced from $1 \mu \mathrm{g}$ of RNA using an anchored-Oligo (dT)18 primer and Transcriptor First Strand cDNA Synthesis Kit (Roche Diagnostics, Basel, Switzerland). The RT reaction was performed in total $20 \mu \mathrm{L}$ at $50^{\circ} \mathrm{C}$ for $60 \mathrm{~min}$, followed by heating at $85^{\circ} \mathrm{C}$ for 5 minutes. The PCR cycling condition was set as follows; an initial denaturing step at $95^{\circ} \mathrm{C}$ for $10 \mathrm{~min}$, and 40 cycles at $95^{\circ} \mathrm{C}$ for $1 \mathrm{~min}, 58$ or $60^{\circ} \mathrm{C}$ for $1 \mathrm{~min}, 72^{\circ} \mathrm{C}$ for $30 \mathrm{~s}$. Primers were as follows: JAZF1, forward 5'-AGCAGTGGAAGCCTTACTCC-3'; SUZ12, reverse 5'-GCTATGAGATTCCGAGTTCGAA G-3'; YWHAE, forward 5' CACTTATCATGCAGTTGT TACGTGAT-3'; FAM22, reverse 5'-GGGCAGAGCC
GTGAACAC-3'; $\beta$-actin as an internal control, forward 5' - TGGCACCACACCTTCTACAA-3' and reverse 5' CCATCACGATGCCAGTGGTA-3'. The same-sized electrophoretic bands indicated amplification of JAZF1SUZ12 transcripts (Fig. 3d), although YWHAE-FAM22 was not detected in all the tumor components.

\section{Discussion}

The $t(7 ; 17)$ translocation resulting in fusion of the JAZF1 gene (on chromosome 7p15) to the SUZ12 gene (on 17q21) represents the most common chromosomal rearrangement in low-grade endometrial stromal tumors, being detected in approximately $30 \%$ of low-grade ESS and $50 \%$ of endometrial stromal nodules tested $[4,5]$. Those tumors share characteristic morphologic features of spindle-cell proliferation similar to those of proliferativephase endometrial stroma, typically lacking significant cytologic atypia and mitotic activity (usually $<5$ counts per $10 \mathrm{HPF}$ ) [2]. The current WHO Classification of Tumors of Female Genital Organs 2014 reintroduced a terminology of high-grade ESS, which was formerly classified into undifferentiated stromal sarcoma and recently reported to harbor the specific YWHAE rearrangement in most cases [6]. High-grade ESS consistently shows uniform cytomorphology, about half of which are biphasic where a round-cell component with mitotic activity (typically $>10$ per $10 \mathrm{HPF}$ ) is admixed with a low-grade-ESS like spindle-cell component commonly with fibrous/fibromyxoid stroma [7]. Immunohistochemically, the round-

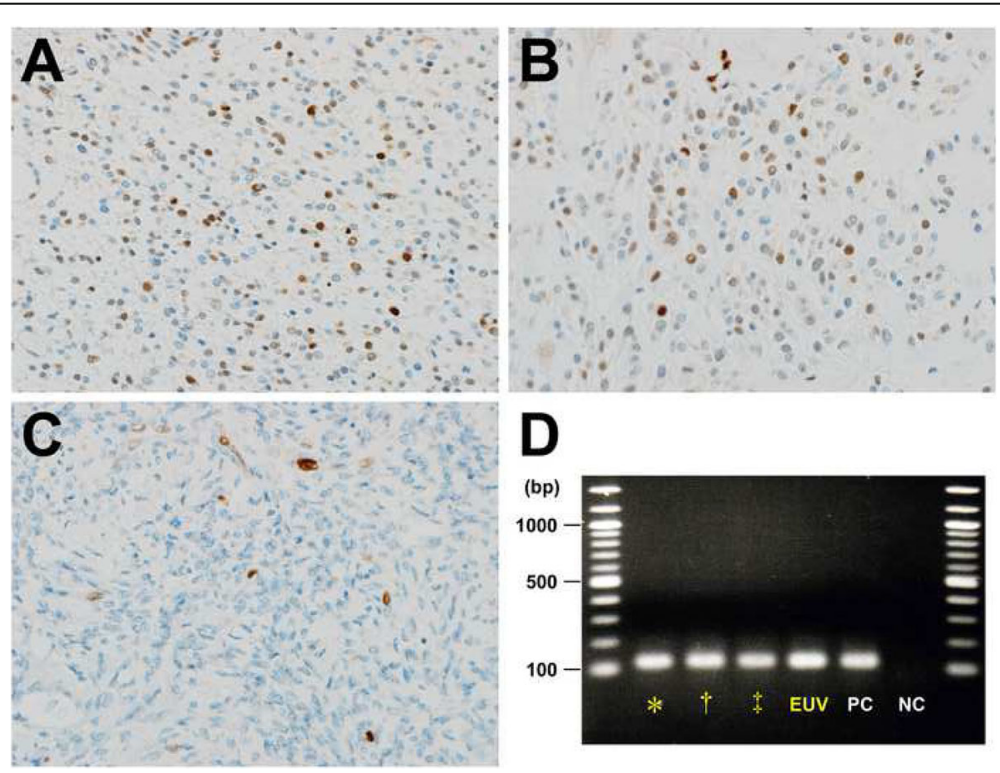

Fig. 3 Cyclin D1 immunostains of the endometrial stromal sarcoma harboring the JAZF1-SUZ12 fusion gene. a and $\mathbf{b} 50 \%$ nuclear staining of tumor cells for Cyclin D1 in the right-anterior (a) and right-posterior (b) components (IHC, $\times 200$ ). c Less than 1\% of tumor cells positive for Cyclin D1 in the fundal component $(\mathrm{IHC}, \times 200)$. $\mathbf{d}$ Reverse transcriptase-polymerase chain reaction demonstrating the JAZF1-SUZ12 chimeric transcripts in the right-anterior $(*)$, right-posterior $(+)$, and the fundal $(\neq)$ tumors and the tumor extension into the extrauterine vein (EUV). PC, positive control; NC, negative control 
cell component usually shows $>70 \%$ cyclin D1 nuclear staining of the tumor cells with homogenous moderate to strong intensity, in contrast to the form of scattered positive cells $(<5 \%)$ in classic $J A Z F 1$ rearranged endometrial stromal tumors [8]. In the present case, the clinically rapid growing component with high FDG uptake demonstrated around $50 \%$ cyclin D1 nuclear immunostaining of neoplastic cells with up to moderate intensity, which indicates the low-grade-ESS like component possibly comprising YWHAE rearranged ESS as Lee et al. reported [8]. This paradoxical heterogeneity in a single JAZF1-SUZ12 ESS with a zonal uptake of FDG has never been documented.

Subserosal masses are much less common in endometrial stromal tumors than in smooth-muscle tumors. The uterine corpus is the most common location for endometrial stromal tumors, although extrauterine locations, specifically the peritoneal surfaces, can be primary sites for the tumor in association with endometriosis, including the ovary, pelvis, fallopian tubes, abdominal cavity, vulva, vagina, bowel wall, urinary bladder, retroperitoneum, and lymph nodes $[9,10]$. Recently, Agaimy et al. reported a man with JAZF1-SUZ12 ESS arising in the paratestis as an extremely rare case [11]. Typically, macroscopic ESS growth patterns feature any combination of (1) intramyometrial nodular masses, (2) an intracavitary polypoid mass, or (3) diffuse myometrial infiltration within expanded uterine walls [2]. The gross appearance of endometrial stromal tumors is occasionally mixed with hemorrhages and necrosis, which may complicate differentiating them from leiomyosarcomas especially when uterine subserosal tumors demonstrate strikingly high FDG uptake. Overt myometrial infiltrations and/or intravascular, worm-like (or "tongue-like") plugs of tumor protruding from intramyometrial or parametrial veins, which are occasionally recognizable on gross examination, are important in distinguishing ESS from leiomyosarcomas [9]. We speculate the two subserosal masses are metastatic from the intramural lesion based on the identical molecular findings and the presence of venous extrauterine permeation.

There were few reports on FDG-PET findings of ESS histologically examined in a large case series; Yamamoto et al. assessed the mean SUV of ${ }^{18}$ F-FDG-PET of 12 uterine sarcomas (8 leiomyosarcomas, 2 high-grade ESS, and 2 low-grade ESS) at $6.1 \pm 3.7$ (range, 1.7-13.5; the highest SUV of ESS was 11.70) [12]. Zhao et al. reported the mean SUV of ${ }^{18} \mathrm{~F}$-FDG-PET of the largest series of 14 uterine malignancies with a mesenchymal component (8 leiomyosarcomas, 3 carcinosarcomas, 1 ESS, and 2 undifferentiated endometrial sarcomas) to be $5.5 \pm 3.1$ (range, 1.8-12.0) [13]. To the best of our knowledge in the literature, SUV of 13.28 in the present case is the highest ${ }^{18} \mathrm{~F}$-FDG uptake in uterine ESS even though there was no radiologic reason for the exceptional uptake, e.g. coexisting histologic inflammation in tumors. In the present case, preoperative levels of serum CA 125 and LDH were slightly elevated, and the former was normalized postoperatively in contrast to the latter staying at a level around $300 \mathrm{U} / \mathrm{L}$. Therefore, CA 125 may be useful for the diagnosis of uterine sarcomas, although this remains nonspecific due to the possibility of stimulated mesothelial cells.

Low-grade ESS is usually indolent with five-year disease specific survival for stages I and II being 90\% compared to $50 \%$ for stages III and IV [14]. Patients with high-grade ESS have earlier and more frequent recurrences (often $<1$ year) and are more likely to die of disease [7], likely to have an intermediate prognosis between low-grade ESS and undifferentiated uterine sarcoma, the latter of which is usually aggressive and exhibits remarkable pleomorphism with no specific translocation pattern $[15,16]$. Since the prognosis of patients with JAZF1-SUZ12 ESS harboring focally high proliferative activity is still unclear, careful follow-up is recommended and future investigations should target this heterogeneity in a single ESS to clarify its prognostic value.

\section{Conclusions}

High FDG uptake on PET is rare in JAZF1-SUZ12 ESS, to which the heterogenous proliferative activity in a single ESS may attribute to this extraordinary radiologic finding. We emphasize that pathologists and clinicians should not exclude the possibility of JAZF1-SUZ12 ESS with a complex growth pattern even when subserosal masses are remarkable, and that molecular analysis is helpful for diagnostic confirmation of JAZF1-SUZ12 ESS with intrauterine metastasis.

\section{Abbreviations \\ CA: Cancer antigen; DWI: Diffusion-weighted images; ESS: Endometrial stromal sarcoma; FDG: Fluorodeoxyglucose; HE: Hematoxylin and eosin; IHC: Immunohistochemistry; LDH: Lactate dehydrogenase; MRI: Magnetic resonance imaging; PET: Positron emission tomography; RT-PCR: Reverse transcriptase polymerase chain reaction; WHO: World Health Organization}

\section{Acknowledgements}

We thank Marla Brunker, from Edanz Group (www.edanzediting.com/ac) for editing a draft of this manuscript.

\section{Authors' contributions}

KF, SN (corresponding author): conception, pathologic diagnosis, and writing of manuscript. RK, CK: pathologic diagnosis and immunohistochemical analysis. MS, MO, TY: molecular oncologic analysis. KN: radiologic analysis. SK: collection of clinical data. SN (last author): revision of manuscript. All authors read and approved the final manuscript prior to submission.

\section{Funding}

Not applicable.

Availability of data and materials

The datasets used and/or analyzed during the current study are available from the corresponding author upon reasonable request. 


\section{Ethics approval and consent to participate}

Not applicable.

\section{Consent for publication}

Written informed consent was obtained from the patient for the publication of this case report. A copy of the consent form is available for review from the editor of this journal.

\section{Competing interests}

The authors declare that they have no competing interests.

\section{Author details}

'Department of Diagnostic Pathology and Cytology, Osaka International Cancer Institute, Osaka, Japan. ${ }^{2}$ Department of Clinical Laboratory, Osaka International Cancer Institute, Osaka, Japan. ${ }^{3}$ Department of Diagnostic and Interventional Radiology, Osaka International Cancer Institute, Osaka, Japan. ${ }^{4}$ Department of Gynecologic Oncology, Osaka International Cancer Institute Osaka, Japan.

Received: 10 July 2019 Accepted: 26 September 2019

Published online: 15 October 2019

\section{References}

1. Silverberg SG, Kurman RJ. Tumors of the uterine corpus: Endometrial stromal tumors. In: Silverberg SG, Kurman RJ, editors. Tumors of the uterine corpus and gestational trophoblastic disease. Atlas of tumor pathology. Third series, Fascicle 3. Washington DC: Armed Forces Institute of Pathology; 1992. p. 91-111.

2. Oliva E, Carcangiu ML, Carinelli S, Ip PP, Loening T, Longacre TA, et al. Tumors of the uterine corpus: endometrial stromal and related tumors. In: Kurman RJ, Carcangiu ML, Herrington CS, Young RH, editors. WHO classification of tumours of female reproductive organs, vol. 6. 4th ed. Lyon: IARC Press; 2014. p. 141-5.

3. Koontz Jl, Soreng AL, Nucci M, Kuo FC, Pauwels P, van Den Berghe H, et al. Frequent fusion of the JAZF1 and JJAZ1 genes in endometrial stromal tumors. Proc Natl Acad Sci U S A. 2001;98:6348-53.

4. Chiang S, Ali R, Melnyk N, McAlpine JN, Huntsman DG, Gilks CB, et al. Frequency of known gene rearrangements in endometrial stromal tumors. Am J Surg Pathol. 2011;35:1364-72.

5. Jakate K, Azimi F, Ali RH, Lee CH, Clarke BA, Rasty G, et al. Endometrial sarcomas: an immunohistochemical and JAZF1 re-arrangement study in low-grade and undifferentiated tumors. Mod Pathol. 2013;26:95-105.

6. Lee CH, Ou WB, Mariño-Enriquez A, Zhu M, Mayeda M, Wang Y, et al. 14-3-3 fusion oncogenes in high-grade endometrial stromal sarcoma. Proc Natl Acad Sci U S A. 2012;109:929-34.

7. Lee $\mathrm{CH}$, Mariño-Enriquez A, Ou W, Zhu M, Ali RH, Chiang S, et al. The clinicopathologic features of YWHAE-FAM22 endometrial stromal sarcomas: a histologically high-grade and clinically aggressive tumor. Am J Surg Pathol. 2012;36:641-53.

8. Lee CH, Ali RH, Rouzbahman M, Marino-Enriquez A, Zhu M, Guo X, et al. Cyclin D1 as a diagnostic immunomarker for endometrial stromal sarcoma with YWHAE-FAM22 rearrangement. Am J Surg Pathol. 2012;36:1562-70.

9. Masand RP, Euscher ED, Deavers MT, Malpica A. Endometrioid stromal sarcoma: a clinicopathologic study of 63 cases. Am J Surg Pathol. 2013;37: 1635-47.

10. Masand RP. Unusual presentations of gynecologic tumors: primary, extrauterine, low-grade endometrioid stromal sarcoma. Arch Pathol Lab Med. 2018;142:536-41.

11. Agaimy A, Moskalev EA, Weisser W, Bach T, Haller F, Hartmann A. Low-grade endometrioid stromal sarcoma of the paratestis: a novel report with molecular confirmation of JAZF1/SUZ12 translocation. Am J Surg Pathol. 2018:42:695-700

12. Yamamoto M, Tsujikawa T, Yamada S, Kurokawa T, Shinagawa A, Chino Y, et al. ${ }^{18} \mathrm{~F}-\mathrm{FDG} /{ }^{18} \mathrm{~F}$-FES standardized uptake value ratio determined using PET predicts prognosis in uterine sarcoma. Oncotarget. 2017:8:22581-9.

13. Zhao Z, Yoshida Y, Kurokawa T, Kiyono Y, Mori T, Okazawa H. ${ }^{18} \mathrm{~F}-\mathrm{FES}$ and ${ }^{18}$ F-FDG PET for differential diagnosis and quantitative evaluation of mesenchymal uterine tumors: correlation with immunohistochemical analysis. J Nucl Med. 2013;54:499-506.

14. Abeler VM, Røyne O, Thoresen S, Danielsen HE, Nesland JM, Kristensen GB. Uterine sarcomas in Norway. A histopathological and prognostic survey of a total population from 1970 to 2000 including 419 patients. Histopathology. 2009:54:355-64.

15. Kurihara S, Oda Y, Ohishi Y, Iwasa A, Takahira T, Kaneki E, et al. Endometrial stromal sarcomas and related high-grade sarcomas: immunohistochemical and molecular genetic study of 31 cases. Am J Surg Pathol. 2008:32:1228-38.

16. Tanner EJ, Garg K, Leitao MM Jr, Soslow RA, Hensley ML. High grade undifferentiated uterine sarcoma: surgery, treatment, and survival outcomes. Gynecol Oncol. 2012;127:27-31.

\section{Publisher's Note}

Springer Nature remains neutral with regard to jurisdictional claims in published maps and institutional affiliations.
Ready to submit your research? Choose BMC and benefit from:

- fast, convenient online submission

- thorough peer review by experienced researchers in your field

- rapid publication on acceptance

- support for research data, including large and complex data types

- gold Open Access which fosters wider collaboration and increased citations

- maximum visibility for your research: over $100 \mathrm{M}$ website views per year

At BMC, research is always in progress.

Learn more biomedcentral.com/submissions 